\title{
A Red Skin Nodule
}

\section{Baroni Adone*}

Dermatology and Venereology, University of Campania "Luigi Vanvitelli", Italy

"Corresponding author: Baroni Adone, Dermatology and Venereology, University of Campania "Luigi Vanvitelli", Italy, Tel: +390815666833; E-mail: adone.baroni@gmail.com

Received date: January 02, 2018; Accepted date: January 05, 2018; Published date: January 08, 2018

Copyright: $\odot 2018$ Adone B. This is an open-access article distributed under the terms of the Creative Commons Attribution License, which permits unrestricted use, distribution, and reproduction in any medium, provided the original author and source are credited.

\section{Image Article}

A 78-year-old woman presented with a 2-month-history of a cutaneous red nodule on her left leg (Figure 1). Differential diagnosis of nodular red lesions includes dermal nevus, pyogenic granuloma, angioma, amelanotic melanoma, eccrine poroma, Kaposi's sarcoma, skin metastasis etc.

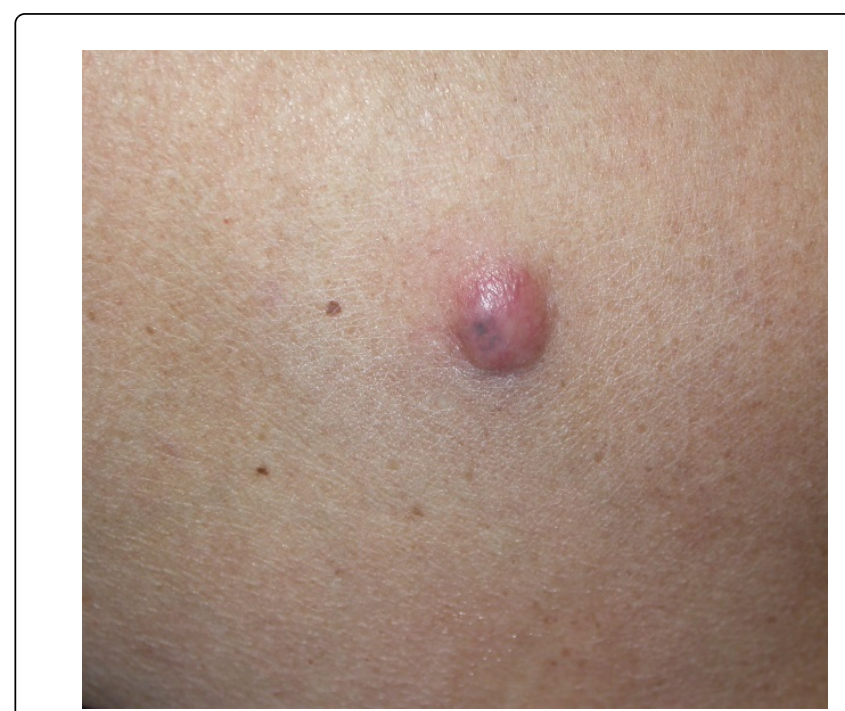

Figure 1: Red Skin Nodule of the left leg

Dermoscopy of the lesion showed irregular diffuse pigmentation, grey-blue area with peppering and red dots (Figure 2). Clinical and dermoscopic features were compatible with diagnosis of nodular amelanotic melanoma. Histologic examination performed on a incisional biopsy showed findings of cutaneous melanoma metastasis. Due to histological data, skin, orogenital mucosae and eyes were examined again, but primary melanoma was not found. So, she made a PET-CT scan with contrast medium that showed FDG accumulation both in subcutaneous skin, in correspondence of the nodule, and in the left colon (Figure 3). Thinking that primary melanoma could be found in the colon, the patient underwent a biopsy of intestinal lesion, that showed histological findings of adenocarcinoma of the colon, and the lack of correlation with cutaneous lesion.

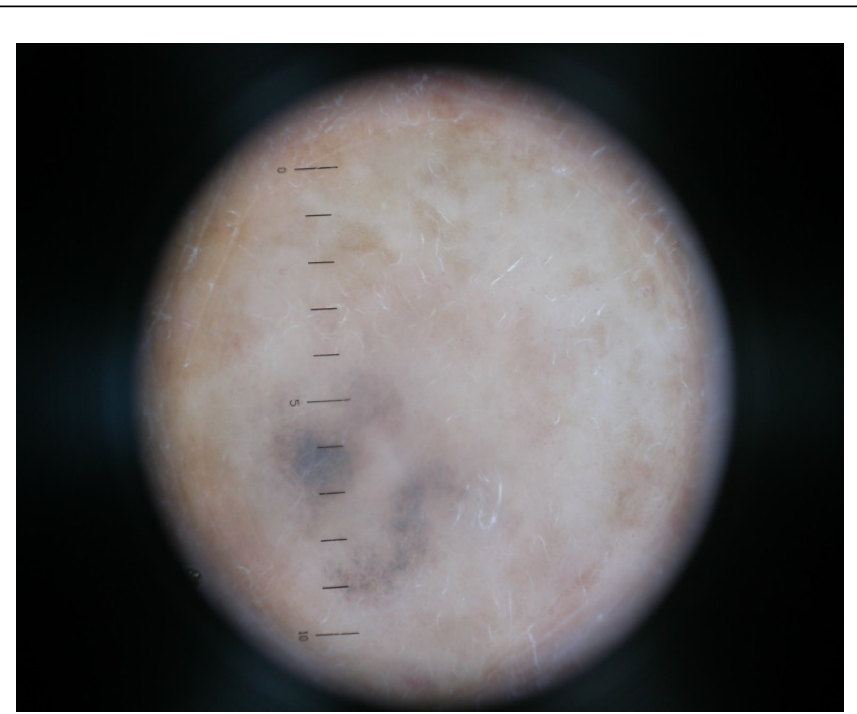

Figure 2: Dermoscopy of the cutaneous red nodule 

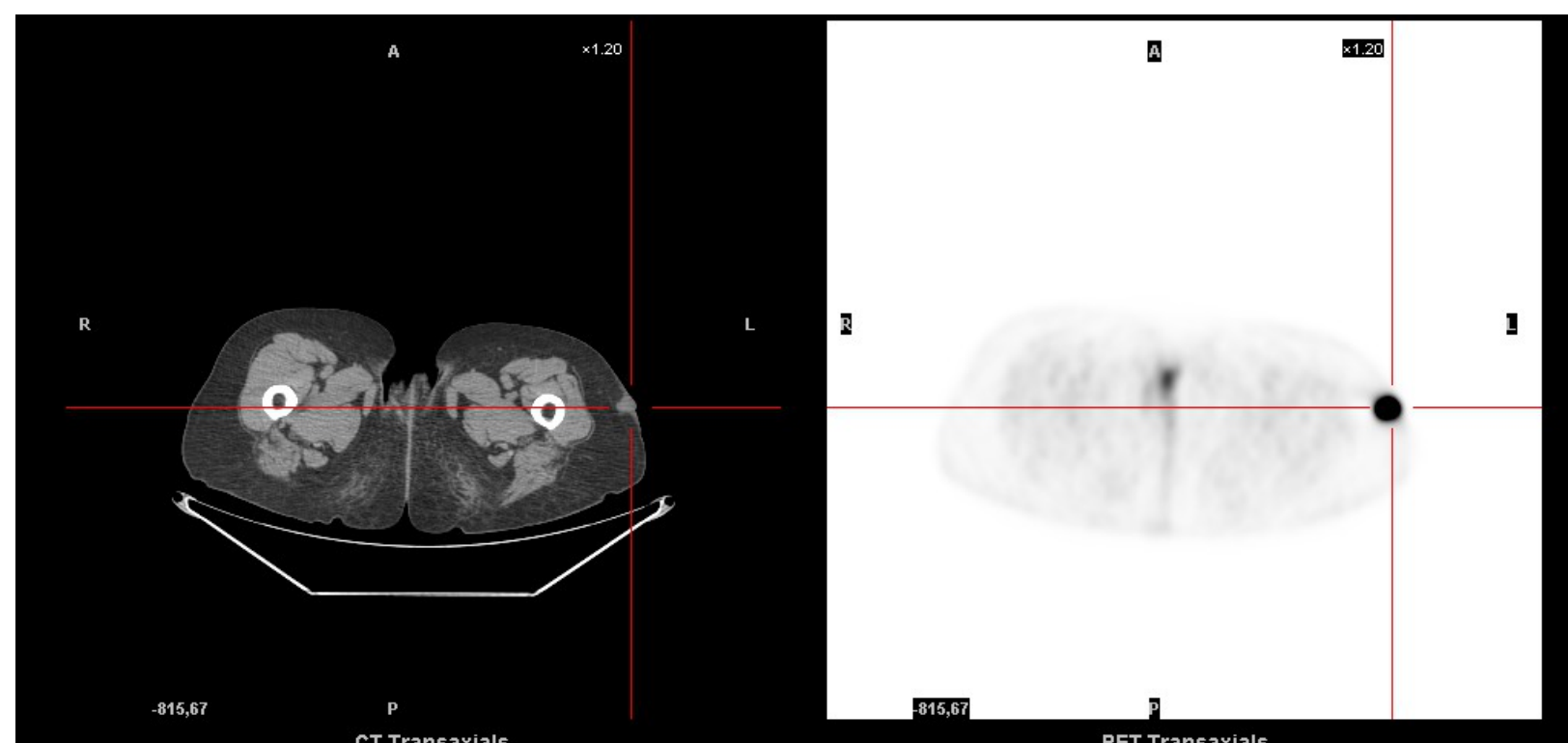

CT Transaxials

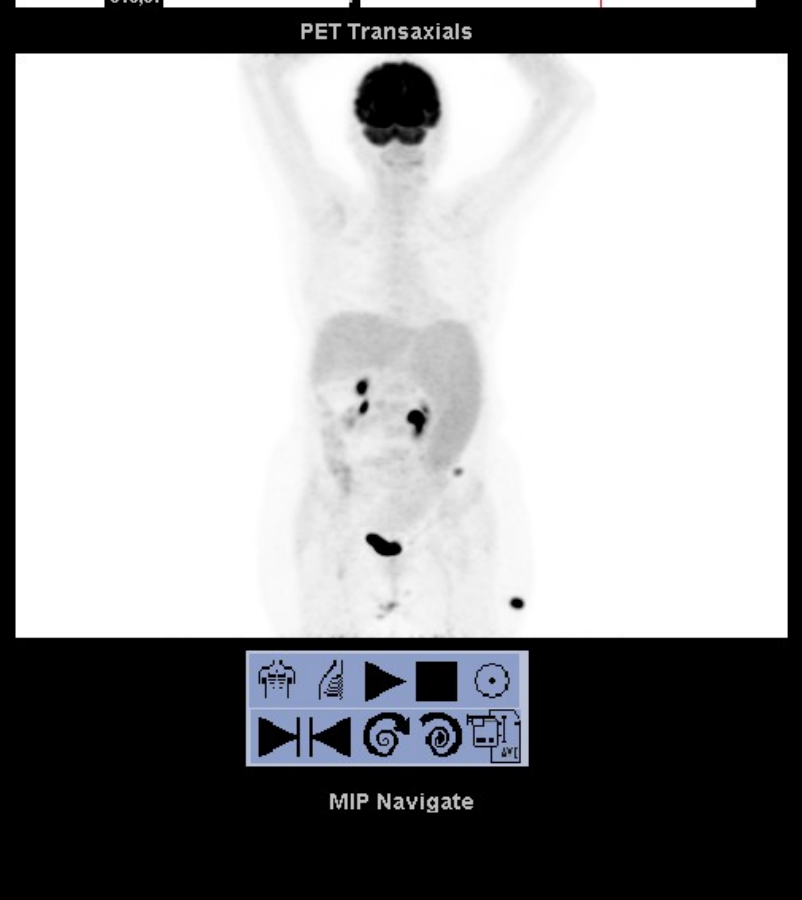

Figure 3: PET-CT scan with contrast medium

After complete excision of the melanoma metastasis, our patient was admitted to Oncology Unit for the inclusion in a therapeutic program, both for melanoma and for synchronous colon adenocarcinoma, but not long afterwards she died for progression of metastatic melanoma.

Melanoma of unknown primary (MUP) is defined as the presence of a histologically confirmed melanoma metastasis in lymph node, internal organ, skin or subcutaneous tissues without history or evidence of primary melanoma [1].

Many hypotheses have been proposed to explain such phenomenon:
- the disappearance of a primary melanoma as a result of immune induced regression after metastasis presentation;

- a previously excised or traumatically removed unidentified melanoma;

- origin of primary melanoma in lymph nodes or in subcutaneous tissues or in internal organs;

- a coexisting unrecognizable melanoma.

The most common event is the immune induced regression of the primary melanoma, through activation of immune-surveillance mechanisms, the recognition of tumour antigens by specific cytotoxic lymphocytes (CTLs) that leads to destruction/regression of the neoplastic cells [2]. 
Citation: Baroni Adone (2018) A Red Skin Nodule. J Integr Oncol 7: i101. doi:10.4172/2329-6771.1000i101

Page 3 of 3

\section{References}

1. Clerico R, Bottoni U, Paolino G, Ambrifi M, Corsetti P, et al. (2012) Melanoma with unknown primary: report and analysis of 24 patients. Med Oncol 29: 2978-2984.
2. Saleh FH, Crotty KA, Hersey P, Menzies SW (2001) Primary melanoma tumour regression associated with an immune response to the tumour-associated antigen melan- $\mathrm{A} /$ MART-1. Int J Cancer 94: 551-557. 